\title{
Enabling Interoperability among Disparate Unmanned Vehicles via Coordinated Command, Control, and Communications Strategies
}

\author{
Joshua Baghdady \\ Applied Research Laboratory \\ University of Hawai' $i$ \\ Honolulu, Hawai'i 96822 \\ United States \\ baghdady@arl.hawaii.edu
}

\author{
A Zachary Trimble \\ RIP Laboratory \\ University of Hawai'i at Mānoa \\ Honolulu, Hawai'i 96822 \\ United States \\ atrimble@hawaii.edu
}

\author{
Manuel Ribeiro \\ Underwater Systems and \\ Technology Laboratory \\ University of Porto \\ Porto, 4200-465 Portugal \\ maribeiro@fe.up.pt
}

\author{
Michael Incze \\ Naval Undersea Warfare Center \\ Division Newport \\ Newport, Rhode Island 02841 \\ United States \\ michael.1.incze@navy.mil
}

\author{
Noah Hafner \\ Applied Research Laboratory \\ University of Hawai' $i$ \\ Honolulu, Hawai' 996822 \\ United States \\ nhafner@arl.hawaii.edu
}

\author{
João Sousa \\ Underwater Systems and \\ Technology Laboratory \\ University of Porto \\ Porto, 4200-465 Portugal \\ jtasso@fe.up.pt
}

\author{
Paulo Dias \\ Underwater Systems and \\ Technology Laboratory \\ University of Porto \\ Porto, 4200-465 Portugal \\ pdias@1sts.pt
}

Raymond Andrade

RIP Laboratory

University of Hawai'i at Mānoa

Honolulu, Hawai‘i 96822

United States

ra2439@hawaii.edu

Ben Jones

Applied Research Laboratory

University of Hawai' $i$

Honolulu, Hawai'i 96822

United States

benjones@arl.hawaii.edu
Keila Lima

Underwater Systems and

Technology Laboratory

University of Porto

Porto, 4200-465 Portugal

keila@1sts.pt

\author{
Maria Costa \\ Underwater Systems and \\ Technology Laboratory \\ University of Porto \\ Porto, 4200-465 Portugal \\ mariacosta@fe.up.pt
}

Margo Edwards

Applied Research Laboratory

University of Hawai' $i$

Honolulu, Hawai'i 96822

United States

margo@arl.hawaii.edu

\begin{abstract}
Although unmanned and autonomous vehicles are continuing to rise in popularity, the lack of interoperability between disparate platforms remains an impediment to multivehicle, multi-domain missions. To address this problem, described herein is a one-year, three-phase research and development effort conducted jointly by researchers from the Applied Research Laboratory at the University of Hawaii, the RIP Laboratory at the University of Hawaii at Manoa, the Naval Undersea Warfare Center Division Newport, and the Underwater Systems and Technology Laboratory at the University of Porto. The focus of this effort was to investigate a Cross Domain Command, Control, and Communications paradigm for heterogenous unmanned vehicle operations that connects platforms across manufacturers, architectures, and interfaces (i.e. air, sea surface, and subsea).
\end{abstract}

Keywords-autonomous underwater vehicles, unmanned underwater vehicles, unmanned surface vehicles, unmanned aerial vehicles, seabed communications, radio frequency communications, acoustic communications, open source software, standards, interoperability

\section{Motivation}

Unmanned and autonomous vehicles (UxVs) have risen sharply in popularity in recent years as component technologies have become more sophisticated and less expensive. Also on the rise is the diversity of $\mathrm{UxV}$ applications, as engineers and developers continue to imagine new ways to put these systems to work [1-7]. Whether slated for use on land, in the air, on the sea, or under the sea, there now exist a plethora of very capable platforms designed to assist humans in almost every capacity imaginable [8-13].

As promising as any one vehicle may be, there exists even greater potential in connecting multiple platforms into a network of coordinated UxVs $[14,15]$. Popularly referred to as swarms, such networks can deliver capability enhancements simply unattainable by discrete platforms. This is all the truer when the swarm consists of disparate UxVs, each with differing, but complementary, capabilities [16]. Such coordinated operations can deliver compound benefits in coverage, time, efficiency, and resolution, demonstrating the veracity of Aristotle when he said, "The whole is greater than the sum of its parts".

Although it is clear that there is much to be gained from collaborative $\mathrm{UxV}$ missions, the interoperability of platforms developed by different manufacturers remains an engineering challenge relegated to the realm of independent research and development (R\&D). Among vehicle manufacturers, greater priority is usually instead placed on perfecting discrete system components, and communications are too often limited to manufacturer-specific intranets governed by proprietary protocols. While this approach may suffice commercial interests in the industrial sector, it overlooks the clear advantages to be gained from true cross-platform cooperation $[17,18]$. 
To address these challenges, researchers from the Applied Research Laboratory at the University of Hawai' $i$ (ARL at UH), the RIP Laboratory at the University of Hawai' ${ }^{i}$ at Mānoa, the Naval Undersea Warfare Center Division Newport (NUWCDIVNPT), and the Underwater Systems and Technology Laboratory at the University of Porto (LSTS) combined efforts in 2019 for a one-year, three-phase R\&D program targeted at UxV interoperability. To reflect the desire to coordinate operations not only across vehicle manufacturers but also across environmental interfaces (i.e. air, sea surface, and subsea), the program adopted the following name: Cross Domain Command, Control, and Communications for Unmanned Systems (CDC3). In the sections that follow, CDC3 2019 is described. Section II gives a description of the participating organizations and the platforms that each contributed to the three phases of CDC3. Section III describes the implementation of $\mathrm{CDC} 3$, and Section IV provides the experimental routines. Section V lends the discussion, and Section VI presents the conclusions.

\section{PARTiCIPANTS \& PlatForms}

In late 2018, NUWCDIVNPT approached LSTS and the ARL at UH to propose a series of joint R\&D efforts intended to advance the capability of distributed control of networked UxVs. This objective and the proposed partners weren't concocted overnight, but rather coalesced from years of previous efforts towards interoperability. It is worth noting that the CDC3 team of 2019 was truly a coalition of the willing in that there was no formal tasking from any sponsoring agency to support the effort. Rather, each organization contributed their own internal research and development (IRAD) funds to advance the state of the art in this area.

In addition to the three primary partners, the RIP Laboratory at UH Mānoa and L3Harris OceanServer both provided crucial vehicle support throughout CDC3 2019. The RIP Laboratory supplemented the ARL at UH by bringing and operating one of their surface platforms at each phase, and L3Harris OceanServer lent an unmanned underwater vehicle (UUV) for each phase and provided support throughout the effort.

With the team thus established, plans were next formed to have each primary partner host one of the three phases of the overall effort. Each phase would provide the team an opportunity to work together in person and field five $\mathrm{UxVs}$ for collaborative testing and evaluation (T\&E). The first phase of CDC3 2019 was hosted in April by the ARL at UH and was held at Moku o Lo'e, commonly known as Coconut Island, on the site of the Hawai' $i$ Institute of Marine Biology in the protected waters of Kāne'ohe Bay on O'ahu's windward side.

To this first phase, the ARL at UH brought their own Seabed Node, a static, seafloor-mounted platform developed specifically for inclusion in the CDC3 network. For a photo of this platform, see Fig. 1. The RIP Laboratory contributed one of their own small unmanned surface vehicles (USVs), affectionally referred to as MRUH (pronounced "Mister UH") see Fig. 2. L3Harris OceanServer shared one of their own IVER3 UUVs (Fig. 3) and an operator, and LSTS provided their own Light Autonomous Underwater Vehicle (LAUV) and Titan unmanned aerial vehicle (UAV) (Figs. 4 and 5, respectively).

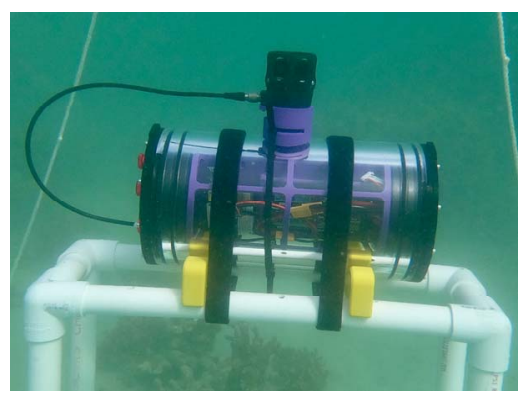

Fig. 1. The ARL at UH's Seabed Node.

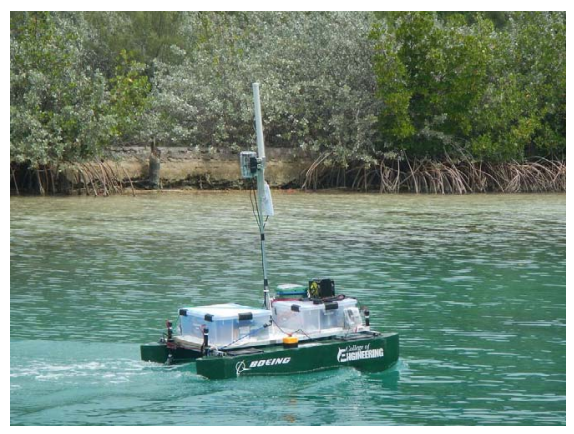

Fig. 2. The RIP Laboratory's MRUH.

The second phase of CDC3 was hosted by NUWCDIVNPT in August and was held at the Narragansett Bay Test Facility in conjunction with the 2019 Advanced Naval Technology Exercise (ANTX). This time, in place of MRUH, the RIP Laboratory brought their Wave Adaptive Modular Vessel (WAM-V) 16 Autonomous Surface Vessel (ASV) - see Fig. 6. Manufactured by Marine Advanced Robotics, Inc., the WAM$\mathrm{V}$ is an impressive platform designed to operate in high sea states while maintaining payload stability. Apart from this upgrade, the vehicles at ANTX were the same as those utilized in phase one.

The third and final phase of $\mathrm{CDC} 3$ was jointly hosted by LSTS, the Portuguese Navy, and the NATO Science and Technology Organization (STO) Centre for Maritime Research and Experimentation (CMRE). It took place in September and was held in Tróia, Portugal in conjunction with the 2019 NATO (Maritime Unmanned Systems) Recognized Environmental Picture Atlantic Exercise [REP(MUS)]. All five of the vehicles tested at ANTX were brought to Portugal for participation in REP(MUS).

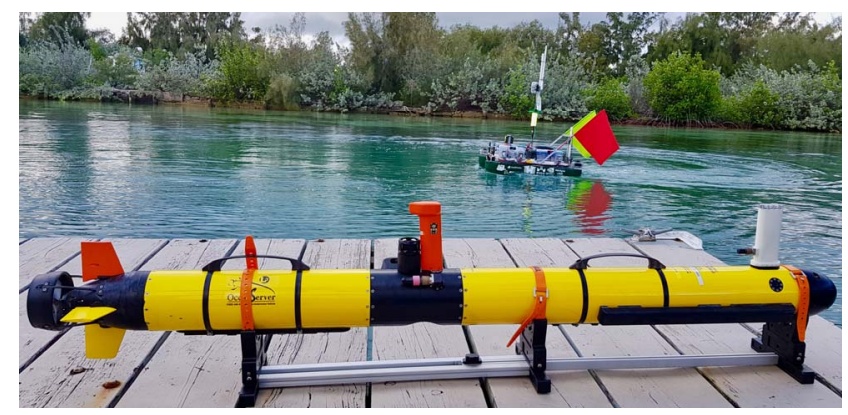

Fig. 3. L3Harris OceanServer's IVER3. 


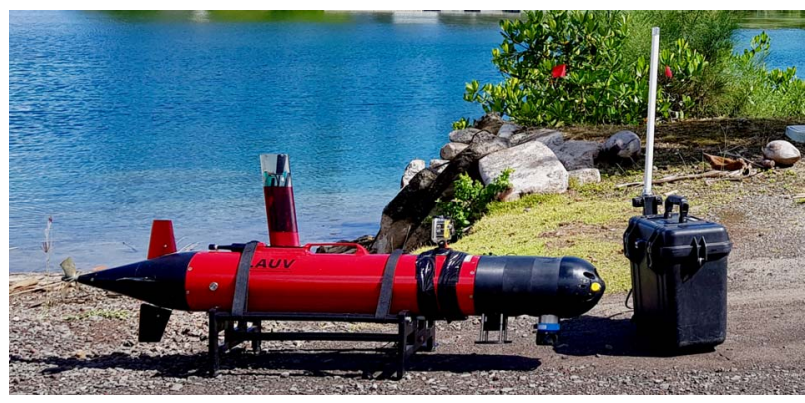

Fig. 4. LSTS' LAUV.

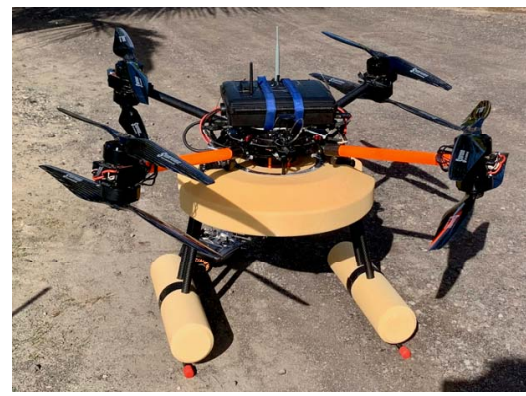

Fig. 5. LSTS' Titan.

\section{IMPLEMENTATION}

Interoperability does not happen by accident, but rather comes as a result of standardization $[19,20]$. In order to enable interoperability among the five disparate $\mathrm{UxVs}$ at $\mathrm{CDC} 3$, they all needed to speak the same language. Additionally, since the goal was to maximize participation in the network in a platformagnostic and license-free manner, open-source software stacks based on publicly-available standards were utilized where possible. In the case of the IVER3, the LAUV, and the Titan, this drove the need for parallel computing architectures on the vehicles: one in the "back seat" to host the open-source, standardized routines, and one in the "front seat" responsible for the physical behavior of the platform, with a logical bridge established between them for communications [21]. In the case of the USV, there was no digital link between the front seat controller and the back seat CDC3 architecture. This eased the transition from MRUH to WAM-V between phases one and two. Since the Seabed Node was a static installation, it did not have a front seat controller.

Hand-in-hand with maximizing vehicle diversity in the network is minimizing common hardware requirements. In the CDC3 2019 paradigm, only one component was universally adopted: the Blueprint Subsea SeaTrac acoustic modem. While there does now exist a digital acoustic communications standard [22], it is very young and most commercial modem manufacturers do not yet support it. In the meantime, the SeaTrac modems provided a common acoustic communications physical layer upon which the remainder of the network could be constructed. Over this channel were sent messages using the LSTS toolchain's Inter-Module Communication Protocol, or IMC [23]. These messages were managed by the LSTS toolchain's DUNE Unified Navigation Environment [23] installed on the back seat of each UxV. Co-hosted alongside DUNE was the Oxford Robotics Institute's Mission Oriented

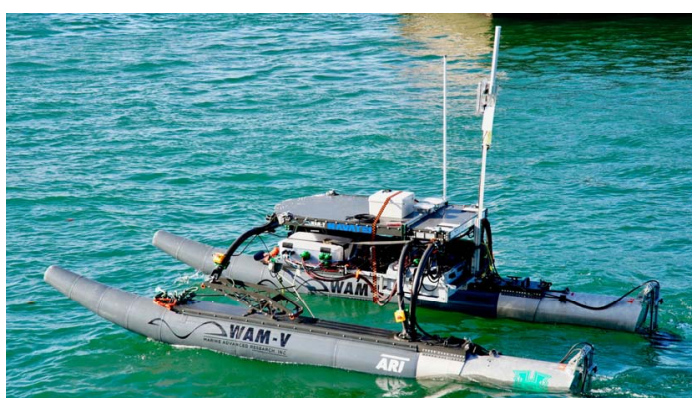

Fig. 6. The RIP Laboratory's WAM-V.

Operating Suite (MOOS) [24], along with its Interval Programming (IvP) extension developed and managed by the Massachusetts Institute of Technology (MIT) [25]. MOOS-IvP served as the primary interface for $\mathrm{CDC} 3$ networking, with MOOS applications constructed for each desired vehicle task.

To enable data exfiltration, NUWCDIVNPT developed a subset of MOOS applications linking MOOS-IvP with the Society of Automotive Engineers' Joint Architecture for Unmanned Systems (SAE-JAUS) standards for communications [26]. This allowed important information from each vehicle to be viewed at a ship- or shore-based ground control station (GCS) whenever the vehicle and the GCS were within radio frequency $(\mathrm{RF})$ communications range. Laptops at the GCS were equipped with the Control Station Human Machine Interface (CaSHMI) from the US Office of Naval Research (ONR) [27] to receive the SAE-JAUS messages, and the LSTS toolchain's NEPTUS Command and Control Software [23] to communicate with DUNE on each vehicle over IMC.

\section{EXPERIMENTAL ROUTINES}

In order to test the CDC3 architecture, four in-water events were designed. Event 1 investigated relaying data from a forward-deployed UUV (both the IVER3 and the LAUV, in turn) back to the GCS via the Titan UAV. The Titan would bridge the air-water interface near the forward-deployed location of the UUV by landing on the water surface. There, it would initiate a data exchange with the UUV via acoustic communications before taking off from the water surface and returning to the GCS, where it would offload the data collected from the UUV over RF communications.

Event 2 was conceptually the same as Event 1, the only difference being that in Event 2 the Titan UAV was replaced by the USV.

Event 3 introduced the Seabed Node and investigated exchanging data between it and the UAV, the USV, and the UUVs in turn. Thereafter, all mobile platforms were to return to the GCS and offload the data collected from the Seabed Node.

Event 4 was designed to combine the demonstrated capabilities of Events 1-3 and extend the roles of the vehicles in an end-to-end operational context. Critical to this Event were networked data exfiltration from forward-deployed mobile and static undersea components without surface intervals or recovery, and the dynamic re-tasking of these components without scheduled communication times and without the use of RF transmissions. In this event, a UUV on an assigned survey 
mission was to be interrupted by higher priority tasking delivered by either the UAV or the USV. This tasking involved rendezvous with the Seabed Node for data sharing and alerting the Seabed Node to initiate a new operational state. The Seabed Node was to then re-task the UUV to perform a new survey mission in an area nearby the original surface asset rendezvous point. Once the UUV had reached this new area and had begun its second survey, the UAV or USV was to again rendezvous with it to exfiltrate the data collected from both the survey missions and the Seabed Node. The surface asset was to subsequently re-task the UUV a final time, sending it to a surface park point for recovery, before returning itself to the GCS for RF offloading of all of the data collected from the mission. For a pictorial representation of Event 4, see Fig. 7.

\section{DISCUSSION}

Given the audacious goals set as described, several challenges were encountered throughout CDC3. Apparent from the beginning was the challenge of coordinating efforts across the ten or eleven time zones which separate Hawai' $i$ from Portugal, depending on the time of year. Software development and integration remained a challenge throughout all three phases of CDC3, as competing protocols such as DUNE and MOOSIvP are not typically implemented simultaneously on the same hardware, and because the vehicles all ran different computer processors. Due to these complications, none of the four Events were executed at phase one. Phase two in Rhode Island provided a much-needed opportunity to diagnose and debug implementation problems, and here the first standalone UxV retasking was demonstrated. In Portugal, new challenges were encountered right away, as the US Navy office responsible for shipping gear from Rhode Island did not properly adjudicate all of the necessary paperwork, and as a result the shipments were delayed several days. When they did finally arrive, the physical T\&E location in the waters of the Sado River introduced additional headaches in the form of strong tidal currents, often in excess of 5 knots between slack tides. At their strongest, these currents precluded UxV operations. Indeed, during the last days of testing at REP(MUS), Event 4 was delayed when the LAUV, having been re-tasked by the Seabed Node to return for further

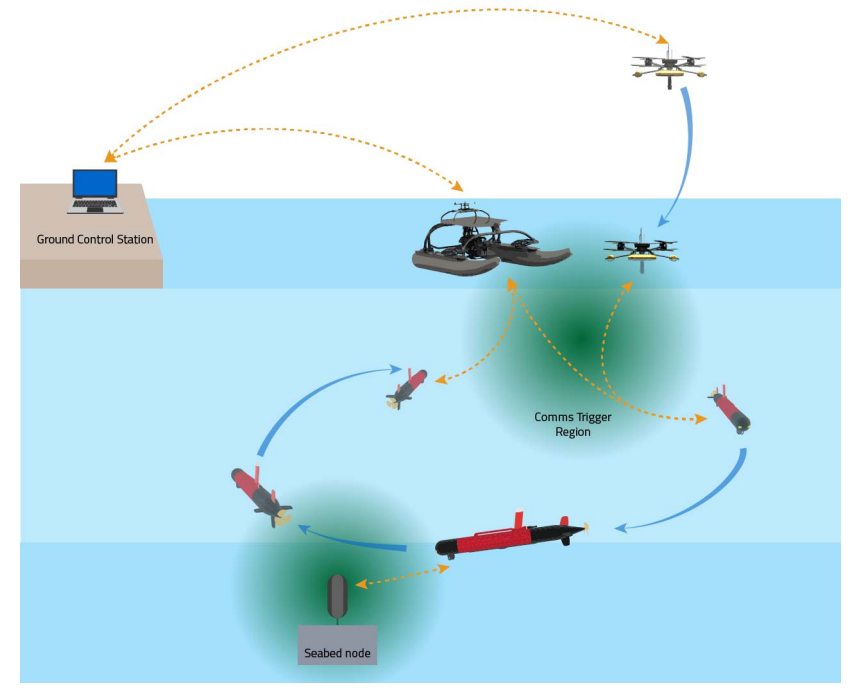

Fig. 7. Event 4. communications with a surface asset, was unable to act on this command because the return route was upcurrent of the Seabed Node. Additionally at REP(MUS), the IVER3 was malfunctioning due to a problem with sending commands from the back seat to the front seat, and the Titan RF hardware was insufficient to support mission execution.

In spite of all of the challenges, final Event 4 was successfully demonstrated on the range at REP(MUS) between the WAM-V, the LAUV, the Seabed Node, and the GCS. This demonstration proved the efficacy of using open-source standards to connect disparate platforms and paved the way for further expansion of this approach in follow-on exercises.

\section{CONCLUSIONS}

In conclusion, the interoperability and seamless networked cooperation of disparate, multi-domain $\mathrm{UxV}$ fleets remains a very promising endeavor with clear payoffs in mission effectiveness and efficiency. The efforts integrated in CDC3 2019 demonstrated the art of the possible in this area, but there is much more to be done. Of pertinent interest for future development is moving away from using a single acoustic modem manufacturer and integrating the new JANUS standard alluded to in Section III [28]. Also of interest is incorporating novel communications techniques, such as optical methods, to further widen the capabilities of the CDC3 network [29]. Lastly, the more is truly the merrier with respect to vehicle integration, and the more diverse processors, platforms, and objectives that can be tested will only serve to make the CDC3 architecture more robust, efficient, and capable of executing a plethora of missions [30].

\section{ACKNOWLEDGMENT}

The authors wish to thank the hosting organizations of each of the three phases of CDC3 2019 for their extensive logistical support. For the first phase, this was the Hawai' $i$ Institute of Marine Biology; special thanks are due to the facilities team for assisting with gear transport to and from Moku o Lo'e, and to the housing team for arranging on-island dormitories for the visiting researchers. For the second phase, this was the staff of NUWCDIVNPT's Narragansett Bay Test Facility, who provided manned dive boats to support the on-water activities. For the third phase, this was the Portuguese Navy, who worked tirelessly throughout REP(MUS) to coordinate activities among nine ships and over $50 \mathrm{UxVs}$ representing more than 10 private companies and the navies of seven NATO nations. Special thanks are due to the Portuguese Navy divers of dive team three, who rapidly and flexibly manned small boats to support CDC3 activities.

\section{REFERENCES}

[1] R. Vicen-Bueno, G. Cimino, D. Cecchi, and B. Garau, "Near real-time scientific data provision from unmanned underwater gliders to remote operational sites," OCEANS 2019 - Marseille, Marseille, France, 2019, pp. 1-9, doi: 10.1109/OCEANSE.2019.8867305.

[2] J. Sánchez-García, D. G. Reina, and S. L. Toral, "A distributed PSO-based exploration algorithm for a UAV network assisting a disaster scenario," Future Gener. Comput. Syst., vol. 90, pp. 129-148, Jan. 2019.

[3] D. Bein, W. Bein, A. Karki, and B. B. Madan, "Optimizing border patrol operations using unmanned aerial vehicles," Proc. 12 ${ }^{\text {th }}$ Int. Conf. Inf. Technol.-Generations, pp. 479-484, Apr. 2015. 
[4] M. L. Incze, "Designing for autonomy in unmanned underwater vehicles," 2012 Oceans, Hampton Roads, VA, 2012, pp. 1-9, doi: 10.1109/OCEANS.2012.6405119.

[5] Y. Liu, Z. Liu, J. Shi, G. Wu, and C. Chen, "Optimization of base location and patrol routes for unmanned aerial vehicles in border intelligence, surveillance, and reconnaissance," J. Adv. Transp., vol. 2019, Jan. 2019.

[6] J. Birch et al., "Autonomous targeted sampling of the deep chlorophyll maximum layer in a subtropical North Pacific eddy," OCEANS 2018 MTS/IEEE Charleston, Charleston, SC, 2018, pp. 1-5, doi: 10.1109/OCEANS.2018.8604898.

[7] Y. Cai et al., "Complementary use of glider data, altimeter for exploring vertical structure of mesoscale eddies in the New England coast," OCEANS 2019 - Marseille, Marseille, France, 2019, pp. 1-7, doi: 10.1109/OCEANSE.2019.8867246.

[8] S. A. Vollgger and A. R. Cruden, "Mapping folds and fractures in basement and cover rocks using UAV photogrammetry, Cape Liptrap and Cape Paterson, Victoria, Australia," J. Struct. Geol., vol. 85, pp. 168-187, Apr. 2016.

[9] T. Lukaczyk, T. Bieri, J. T. de Sousa, J. Levy, and P. A. McGillivary, "Unmanned aircraft as mobile components of Ocean Observing Systems for management of marine resources," OCEANS 2016 MTS/IEEE Monterey, Monterey, CA, 2016, pp. 1-7, doi: 10.1109/OCEANS.2016.7761485.

[10] P. Lottes, R. Khanna, J. Pfeifer, R. Siegwart, and C. Stachniss, "UAVbased crop and weed classification for smart farming," Proc. IEEE Int. Conf. Robot. Automat. (ICRA), pp. 3024-3031, May/Jun. 2017.

[11] J. Levy, C. Hunter, T. Lukaczyk, and E. C. Franklin, "Assessing the spatial distribution of coral bleaching using small unmanned aerial systems," Coral Reefs, vol. 37, pp. 373-387, Feb. 2018.

[12] M. L. Incze, "Demonstrated performance of economical, man-portable AUVs in multi-vehicle coastal surveys," OCEANS 2010 MTS/IEEE SEATTLE, Seattle, WA, 2010, pp. 1-7, doi: 10.1109/OCEANS.2010.5664344.

[13] C. Barrera et al., "Expanding ocean-observations in the Macaronesia region with autonomous mobile platforms," OCEANS 2019 - Marseille, Marseille, France, 2019, pp. 1-5, doi: 10.1109/OCEANSE.2019.8867517.

[14] L. T. Parker, M. J. Ferro, and S. R. Sideleau, "Testing multi-agent autonomy in complex communications environments," OCEANS 2016 MTS/IEEE Monterey, Monterey, CA, 2016, pp. 1-5, doi: 10.1109/OCEANS.2016.7761192.

[15] D. Portugal, C. Pippin, R. P. Rocha, and H. Christensen, "Finding optimal routes for multi-robot patrolling in generic graphs," 2014 IEEE/RSJ International Conference on Intelligent Robots and Systems, Chicago, IL, 2014, pp. 363-369, doi: 10.1109/IROS.2014.6942585.

[16] M. Novitsky, C. Pippin, T. R. Collins, T. R. Balch, and M. E. West, "Bioinspired multi-robot communication through behavior recognition," 2012 IEEE International Conference on Robotics and Biomimetics (ROBIO), Guangzhou, 2012, pp. 771-776, doi: 10.1109/ROBIO.2012.6491061.

[17] X. Xiao, J. Dufek, T. Woodbury, and R. Murphy, "UAV assisted USV visual navigation for marine mass casualty incident response," 2017
IEEE/RSJ International Conference on Intelligent Robots and Systems (IROS), Vancouver, BC, 2017, pp. 6105-6110, doi: 10.1109/IROS.2017.8206510.

[18] C. A. Harris et al., "Oceanids C2: An integrated command, control, and data infrastructure for the over-the-horizon operation of marine autonomous systems," Front. Mar. Sci., vol. 7, art. 397, Jun. 2020, doi: 10.3389/fmars.2020.00397.

[19] D. Serrano, "Key initiatives for interoperability through standardization applicability to small unmanned vehicles," The NATO STO Lecture Series, vol. STO-EN-SCI-271, art. 1, pp. 1-14, May 2015.

[20] M. M. Marques, "STANAG 4586 - Standard interfaces of UAV control system (UCS) for NATO UAV interoperability," The NATO STO Lecture Series, vol. STO-EN-SCI-271, art. 3, pp. 1-14, May 2015.

[21] A. V. Rodrigues, R. S. Carapau, M. M. Marques, V. Lobo, and F. Coito, "Unmanned systems interoperability in military maritime operations: MAVLink to STANAG 4586 bridge," OCEANS 2017 - Aberdeen, Aberdeen, 2017, pp. 1-5, doi: 10.1109/OCEANSE.2017.8084866.

[22] J. Potter, J. Alves, D. Green, G. Zappa, I. Nissen, and K. McCoy, “The JANUS underwater communications standard," 2014 Underwater Communications and Networking (UComms), Sestri Levante, 2014, pp. 1-4, doi: 10.1109/UComms.2014.7017134

[23] J. Pinto, P. S. Dias, R. Martins, J. Fortuna, E. Marques, and J. Sousa, "The LSTS toolchain for networked vehicle systems," 2013 MTS/IEEE OCEANS - Bergen, Bergen, 2013, pp. 1-9, doi: 10.1109/OCEANSBergen.2013.6608148.

[24] P. M. Newman, "MOOS - Mission Oriented Operating Suite," OUEL Report, no. 2299/08, 2008.

[25] M. R. Benjamin, H. Schmidt, P. M. Newman, J. J. Leonard, "Nested autonomy for unmanned marine vehicles with MOOS-IvP," J. Field Robotics, vol. 27, no. 6, pp. 834-875, 2010, doi: 10.1002/rob.20370.

[26] M. L. Incze, S. R. Sideleau, C. Gagner, and C. A. Pippin, "Communication and collaboration of heterogenous unmanned systems using the Joint Architecture for Unmanned Systems (JAUS) standards," OCEANS 2015 - Genova, Genoa, 2015, pp. 1-6, doi: 10.1109/OCEANSGenova.2015.7271613.

[27] S. R. Sideleau, D. Powell, L. M. Ewart, J. G. Morrison, "Control Station Human Machine Interface (CaSHMI),” NUWC Newport, Oct. 2016.

[28] J. Alves et al., "Moving JANUS forward: a look into the future of underwater communications interoperability," OCEANS 2016 MTS/IEEE Monterey, Monterey, CA, 2016, pp. 1-6, doi: 10.1109/OCEANS.2016.7761094.

[29] P. A. McGillivary, V. Chirayath, and J. Baghdady, "Use of multi-spectral high repetition rate LED systems for high bandwidth underwater optical communications, and communications to surface and aerial systems, "2018 Fourth Underwater Communications and Networking Conference (UComms), Lerici, 2018, pp. 1-5, doi: 10.1109/UComms.2018.8493228.

[30] M. Pradhan, A. Tiderko, and D. Ota, "Approach towards achieving an interoperable C4ISR infrastructure," 2017 International Conference on Military Technologies (ICMT), Brno, 2017, pp. 375-382, doi: 10.1109/MILTECHS.2017.7988788. 